\title{
Enhanced vaccine control of epidemics in adaptive networks
}

Leah B. Shaw

William \& Mary, lbshaw@wm.edu

Ira B. Schwartz

Follow this and additional works at: https://scholarworks.wm.edu/aspubs

\section{Recommended Citation}

Shaw, L. B., \& Schwartz, I. B. (2010). Enhanced vaccine control of epidemics in adaptive networks.

Physical Review E, 81(4), 046120.

This Article is brought to you for free and open access by the Arts and Sciences at W\&M ScholarWorks. It has been accepted for inclusion in Arts \& Sciences Articles by an authorized administrator of W\&M ScholarWorks. For more information, please contact scholarworks@wm.edu. 


\title{
Enhanced vaccine control of epidemics in adaptive networks
}

\author{
Leah B. Shaw \\ Department of Applied Science, College of William and Mary, Williamsburg, Virginia 23187, USA \\ Ira B. Schwartz \\ Code 6792, Nonlinear Systems Dynamics Section, Plasma Physics Division, \\ US Naval Research Laboratory, Washington, DC 20375, USA \\ (Received 29 June 2009; revised manuscript received 9 March 2010; published 29 April 2010)
}

\begin{abstract}
We study vaccine control for disease spread on an adaptive network modeling disease avoidance behavior. Control is implemented by adding Poisson-distributed vaccination of susceptibles. We show that vaccine control is much more effective in adaptive networks than in static networks due to feedback interaction between the adaptive network rewiring and the vaccine application. When compared to extinction rates in static social networks, we find that the amount of vaccine resources required to sustain similar rates of extinction are as much as two orders of magnitude lower in adaptive networks.
\end{abstract}

DOI: 10.1103/PhysRevE.81.046120

PACS number(s): 89.75.Hc, 87.19.X-

\section{INTRODUCTION}

Modeling the spread of epidemics on static networks is a well-developed field, but recent studies have begun to account for potentially time-varying network topologies. In particular, people may adjust their social behavior in response to the threat of an epidemic. Both a susceptibleinfected-susceptible (SIS) model [1] and susceptibleinfected-recovered-susceptible (SIRS) model [2] have been studied on an adaptive network in which noninfected nodes rewire their links adaptively away from infected neighbors and toward other noninfected nodes. Such adaptation typically increases the epidemic threshold and reduces the number of infectious cases, and new disease dynamics and bifurcations are observed. Similar results are seen when the nodes reconnect to randomly selected nodes elsewhere in the network $[3,4]$. Because vaccines are available for many diseases, it is desirable to examine the interplay between adaptively fluctuating social contacts and vaccination of susceptible individuals.

Almost all diseases exhibit randomness resulting in observed fluctuations, as in [5-10]. As diseases evolve in large populations, there is the possibility of finite time extinction [10-15]. Extinction occurs where the number of infectives becomes so small that there is insufficient transmission to keep the disease in its endemic state [16-18]. Fluctuations cause the disease-free equilibrium (DFE) to be reached in a finite time. Such an extinction process occurs even when the DFE is unstable. Populations based upon adaptive networks further complicate the problem, since social dynamical situations, such as disease avoidance strategies, can cause the endemic and DFE to be bistable [2].

A major characteristic of fluctuation-induced extinction in stochastic models for globally connected large populations is the extinction rate. Viewing disease fade-out as coming from systems far from thermal equilibrium, finite population extinction rate laws have been derived in SIS [15,19] and SIR [20] models. In contrast to vaccine strategies which stabilize the DFE [21-26], periodic pulsed vaccination was generalized to a random Poisson strategy, which exponentially enhances the rate of extinction [27].
Other vaccine strategies have been examined in a variety of static network geometries. Targeting of high degree nodes is widely recognized as more effective than random vaccination, including in scale-free networks [28,29], small world networks [30], and more realistic social network geometries [31]. Because targeting the highest degree nodes requires full knowledge of the network geometry, other strategies based on local knowledge have been developed. For example, vaccinating a random acquaintance of a randomly selected node tends to favor high degree nodes and is again more effective than random vaccination [32]. When vaccine is very limited, outbreaks can be minimized by fragmenting the network via a graph partitioning strategy which requires less vaccine than targeting high degree nodes [33].

Here we will use a random vaccination strategy and find that in conjunction with adaptive rewiring, it is extremely effective. Section II introduces our basic SIS model with vaccination and a corresponding mean-field theory, Sec. III presents results for endemic states and epidemic extinction, Sec. IV shows that the introduction of a recovered node class does not qualitatively alter our results, and Sec. V concludes.

\section{SIV MODEL}

We create a model by modifying an SIS model [1], adding a vaccinated class $(V)$. Individuals are connected in a network, with noninfected nodes rewiring adaptively to reduce connections with infected nodes.

The transition probabilities are as follows. A susceptible node becomes infected with rate $p N_{I, \mathrm{nbr}}$, where $N_{I, \mathrm{nbr}}$ is its number of infected neighbors. An infected node recovers (to susceptible) with rate $r$. Motivated by national immunization days [34], vaccination occurs in Poisson-distributed pulses with frequency $\nu$, and each pulse vaccinates a fraction $A$ of the susceptible nodes. We assume that the vaccine is not permanent, so a vaccinated node becomes susceptible again with rate $q$, the resusceptibility rate.

While the epidemic spreads, the network is rewired adaptively. Rewiring rather than link cutting is considered because we focus on the long term epidemic behavior. 
Although an outbreak could transiently decrease network connectivity in a real social network, long term reductions in average connectivity are unrealistic and difficult to maintain. Because the vaccine wears off, we assume that vaccinated nodes are uncertain of their infection status and thus rewire in the same way as susceptibles. If a link connects a noninfected node to an infected node, that link is rewired with rate $w$ to connect the noninfected node to another randomly selected noninfected node. Self links and multiple links between nodes are excluded.

We simulate the full adaptive network via Monte Carlo simulation in a similar fashion to [2]. Vaccination events occur with average rate $\nu$, and in each event $A N_{S}$ susceptibles are selected randomly for vaccination, where $N_{S}$ is the total number of susceptibles. Results are presented here for $N=10^{4}$ nodes and $K=10^{5}$ links.

We have developed a mean-field theory for the dynamics of nodes and links following [1,2]. $P_{A}$ denotes the probability of a node to be in state $X$, where $X$ is either $S, I$, or $V . P_{X Y}$ denotes the probability that a randomly selected link connects a node in state $X$ to a node in state $Y$. If we assume that the vaccine pulses are applied at the mean frequency $\nu$ and vaccinate a fraction $A$ of the susceptible population, we obtain the following set of deterministic differential equations for the nodes:

$$
\begin{gathered}
\dot{P}_{S}=r P_{I}-p \frac{K}{N} P_{S I}-\nu A P_{S}+q P_{V}, \\
\dot{P}_{I}=p \frac{K}{N} P_{S I}-r P_{I}, \\
\dot{P}_{V}=\nu A P_{S}-q P_{V},
\end{gathered}
$$

and for the links:

$$
\begin{aligned}
& \dot{P}_{S S}=r P_{S I}-2 p \frac{K}{N} \frac{P_{S S} P_{S I}}{P_{S}}-\nu\left(2 A-A^{2}\right) P_{S S}+q P_{S V}+w \frac{P_{S}}{P_{S}+P_{V}} P_{S I}, \\
& \dot{P}_{S I}=2 p \frac{K}{N} \frac{P_{S S} P_{S I}}{P_{S}}-p\left(P_{S I}+\frac{K}{N} \frac{P_{S I}^{2}}{P_{S}}\right)-r P_{S I}+2 r P_{I I} \\
& +q P_{I V}-\nu A P_{S I}-w P_{S I} \text {, } \\
& \dot{P}_{S V}=r P_{I V}-p \frac{K}{N} \frac{P_{S I} P_{S V}}{P_{S}}+2 q P_{V V}-q P_{S V}-\nu A P_{S V} \\
& +2 \nu A(1-A) P_{S S}+w \frac{P_{V}}{P_{S}+P_{V}} P_{S I}+w \frac{P_{S}}{P_{S}+P_{V}} P_{I V}, \\
& \dot{P}_{I I}=p\left(P_{S I}+\frac{K}{N} \frac{P_{S I}^{2}}{P_{S}}\right)-2 r P_{I I}, \\
& \dot{P}_{I V}=p \frac{K}{N} \frac{P_{S I} P_{S V}}{P_{S}}-r P_{I V}-q P_{I V}+\nu A P_{S I}-w P_{I V}, \\
& \dot{P}_{V V}=\nu A P_{S V}-2 q P_{V V}+\nu A^{2} P_{S S}+w \frac{P_{V}}{P_{S}+P_{V}} P_{I V} .
\end{aligned}
$$

Vaccination terms that are second order in the pulse amplitude $A$ appear in the link equations because each pulse can vaccinate multiple susceptibles, so a fraction $A^{2}$ of the SS links are converted directly to $\mathrm{VV}$ links, while a fraction $2 A(1-A)$ of them become SV links. The stochasticity of the vaccine pulses can be incorporated in the mean field by replacing the average pulse frequency $\nu$ by a Poisson-

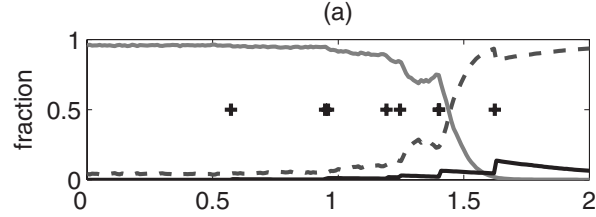

(b)

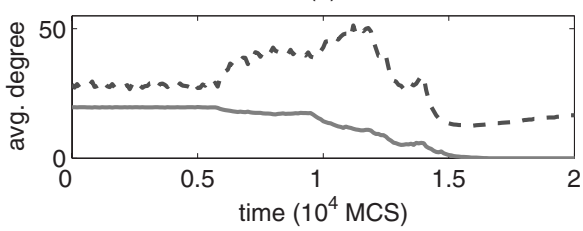

FIG. 1. Sample time series. (a) Node fractions. Solid gray curve: infectives; dashed dark gray curve: susceptibles; solid black curve: vaccinated. "+" symbols indicates times of vaccine pulses. (b) Average degree by node class. Solid gray curve: infectives; dashed dark gray curve: susceptibles. $p=0.003, \quad r=0.002$, $q=0.0002, A=0.1, \nu=0.0005$, and $w=0.04$.

distributed stochastic variable $\eta(t)$ with the appropriate average frequency and vaccination amplitude.

\section{SIV RESULTS}

Sample time series are shown in Fig. 1 for a run in which the epidemic became extinct. Because we use proportional vaccination, as vaccination begins to lower the number of infectives and the number of susceptibles increases, subsequent vaccine pulses vaccinate a larger number of nodes.

We studied the longtime behavior for the case of longer lived endemic states. The deterministic mean-field model predicts stable steady-state dynamics for the static network, even when vaccine is applied. Small regions of oscillatory behavior have been predicted for the adaptive network without vaccination [1], and these regions are predicted to be much larger when vaccination is applied. For the resusceptibility $q$ used here, oscillations are generic [39].

Figures 2(a)-2(d) compare the dependence of the mean infective level on the vaccine frequency for static and adaptive networks [40]. For the full static (Erdős-Rényi) network system [Fig. 2(b)], at each vaccine frequency the steady-state mean infectives were computed over ten network geometries. For the stochastic mean field [Fig. 2(a)], 100 runs were done to extinction $\left(P_{I}<10^{-12}\right)$ and the means of the time series were computed. The mean field and full model are in excellent agreement.

For the full system with rewiring [Fig. 2(d)], infectives were averaged over $8 \times 10^{4}$ MCS for a single adaptive network for the smaller vaccine frequencies $(\nu \leq 0.00015)$, and error bars are the standard deviation. For larger $\nu$ values the oscillations led to rapid die out, so the infectives were instead averaged over 100 runs computed to extinction, where the vaccine was turned on at time 0. Error bars are the standard deviation of all the time points. This averaging includes transients but serves to illustrate the decreasing infective levels and large fluctuations due to oscillations as $\nu$ is increased. The stochastic mean field [Fig. 2(c)] was computed as in Fig. 2(a), and error bars are the standard deviations of the means. 

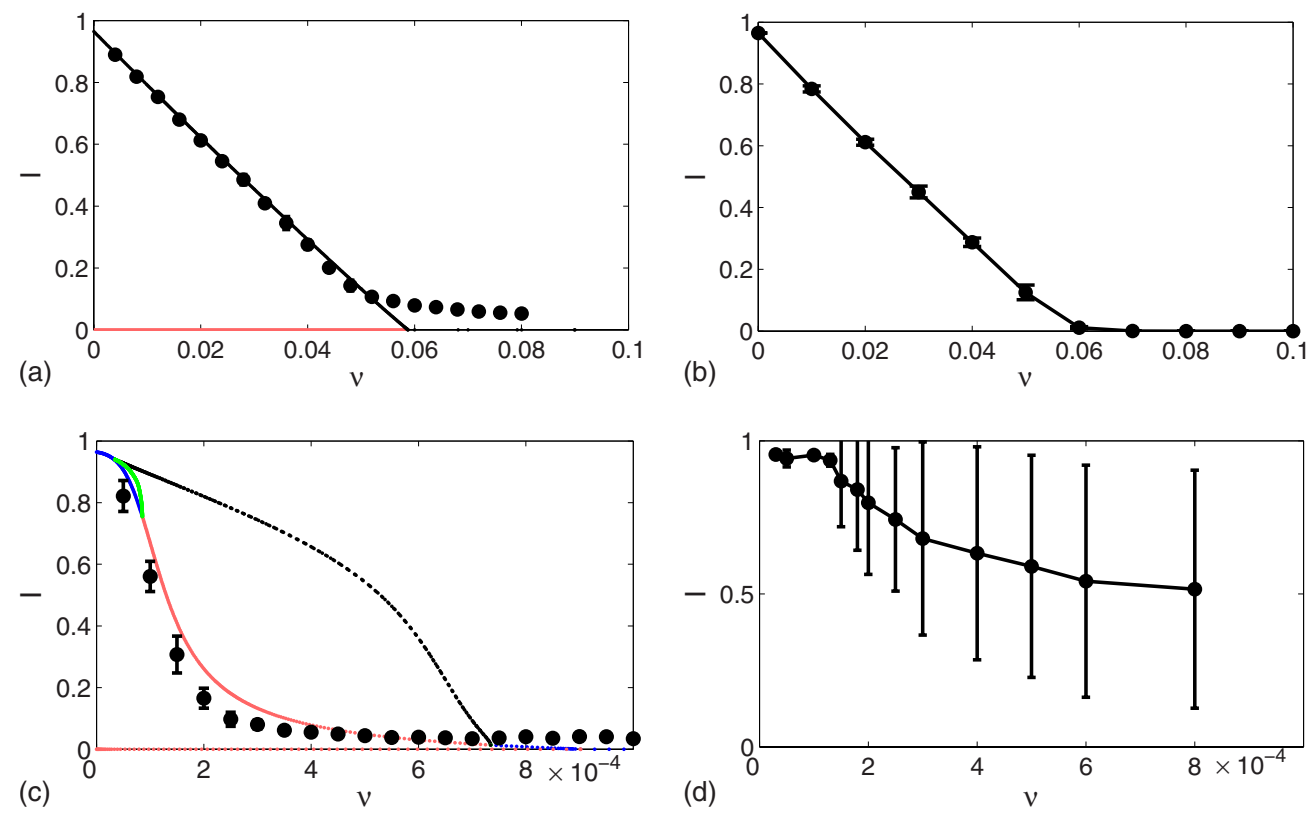

FIG. 2. (Color online) Average infectives versus vaccine frequency $\nu$ for $q=0.0002, r=0.002, p=0.003, A=0.1$. (a) Mean field, $w=0$. Black and red (gray) curves, respectively, are stable and unstable branches of the deterministic mean field. Symbols are stochastic mean field. (b) Full system, $w=0$. Curve is to guide the eyes. (c) Mean field, $w=0.04$. Blue (dark gray) curve: stable steady state, red (medium gray) curve: unstable steady state, black curve: stable periodic orbit, green (light gray) curve: unstable periodic orbit for deterministic model. Symbols are stochastic mean field. (d) Full system, $w=0.04$.

The vaccination frequency required to significantly lower the infected fraction in the adaptive network is much smaller than for the static network due to the interaction of vaccination and rewiring. The mean-field model accurately predicts the order of magnitude of vaccine required and the presence of oscillations.

Figure 3 shows the dependence of the endemic state lifetime on the vaccine frequency for static (Erdős-Rényi) and adaptive networks. About two orders of magnitude less vaccine is needed in the adaptive case to significantly reduce the lifetime of the endemic state. For the full system, each point was obtained by averaging 100 runs for which the initial condition was the vaccine-free steady state and the vaccine was turned on at time zero. Mean-field results were obtained similarly, but the time to extinction was computed using a threshold for $P_{I}$ of $10^{-12}$.

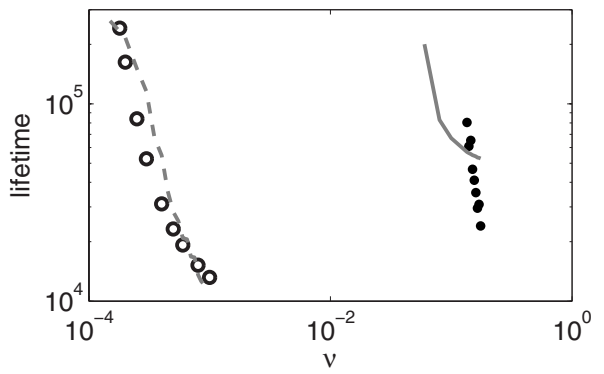

FIG. 3. Dependence of endemic state average lifetime on vaccine frequency $\nu$. Black open circles and dashed gray curve are full system and mean field, respectively, with rewiring $(w=0.04)$. Black closed circles and solid gray curve are full system and mean field, respectively, with no rewiring. $p=0.003, r=0.002, q=0.0002$, and $A=0.1$.
To explain the efficacy of vaccination in the adaptive network, we examine the network structure in more detail, particularly the degree. Steady-state degree distributions for an adaptive network with and without vaccination are shown in Fig. 4. Results without vaccination were obtained similarly to those in [2]. Results with vaccination were computed likewise but averaging over nine runs to obtain better statistics for the vaccinated nodes which are present at very low levels (0.6\% of the nodes). As shown in Fig. 4(a), susceptibles in

(a)

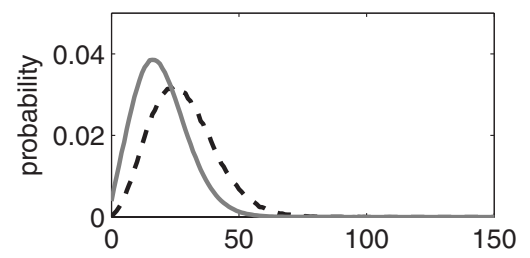

(b)

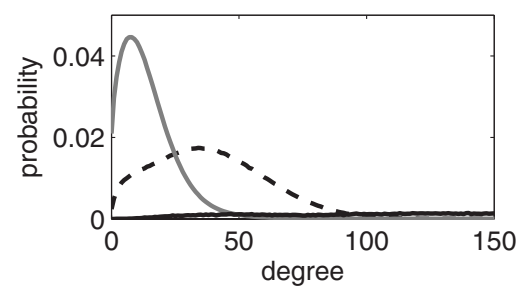

FIG. 4. Degree distributions from Monte Carlo simulation for $p=0.002, r=0.002, w=0.04$. (a) No vaccination. (b) With vaccination: $\nu=0.00015, A=0.1, q=0.0002$. Degree distribution of vaccinated nodes is very broad, extending beyond the figure domain, with an average degree of 579. Solid gray: infectives; dashed: susceptibles; and solid black: vaccinated. 
the adaptive network have a higher average degree than infectives due to rewiring. This is also apparent at the beginning of the degree time series for susceptibles and infectives in Fig. 1(b). Because the susceptibles typically have higher degree, random vaccination of susceptible nodes favors the higher degree nodes in the network and is therefore expected to be effective, as in previous studies of targeted vaccination [28-30]. For a static network, in contrast, the high degree nodes are most likely to be infected and thus will rarely be selected for vaccination.

The vaccination level used in Fig. 4(b) is small enough that it only slightly lowers the number of infected nodes. However, when vaccination occurs the rewiring becomes more effective because of the presence of "safe" nodes to wire to. The average infective degree is substantially decreased, and the susceptible degree is substantially increased. The vaccinated nodes have a broad degree distribution with a very high average degree. For the parameter values in Fig. 4(b), vaccinated nodes participate in links as frequently as susceptibles, even though there are two orders of magnitude fewer vaccinated than susceptibles.

\section{SIRV MODEL}

To connect more closely with real diseases, for which recovery from infection often confers a period of immunity, we have extended our model to an SIRS (susceptibleinfected-recovered-susceptible) model with a vaccinated class. The SIRS dynamics is modeled as in [2], with infection occurring with rate $p N_{I, \text { nbr }}$, recovery with rate $r$, and resusceptibility with rate $q$. As we have described here for the SIV model, we include Poisson-distributed pulsed vaccination of susceptibles with average rate $\nu$, and a fraction $A$ of the susceptibles are vaccinated in each pulse. Immunity from vaccination is assumed to wear off with the same rate $q$ as natural immunity. All links from noninfected to infected nodes can rewire with rate $w$, and a noninfected node chooses its new neighbor at random from all possible noninfected nodes. We performed Monte Carlo simulations for the SIRV model for a system with $N=10^{4}$ nodes and $K=10^{5}$ links, as described above for the SIV model. Figure 5 shows the dependence of the mean infective level on the vaccine frequency for static and adaptive networks. The results in Fig. 5(a) were computed as in Fig. 2(b). The results in Fig. 5(b) were computed similarly to Fig. 2(d), with steady-state averages for $\nu \leq 0.007$ and averages over ten runs computed to extinction for $\nu>0.007$ due to rapid die out. Over an order of magnitude more vaccine is required to drive the epidemic to extinction in the static network case.

\section{CONCLUSIONS AND DISCUSSION}

In summary, we studied the effect of Poisson vaccination on epidemic spread in an adaptive network. Adaptation of the network was examined by modeling the response of individuals in the presence of an epidemic. One of the main goals was to examine the interplay of rewiring of the network and probabilistic vaccination strategies in a new class of adaptive network models which are driven by (a)

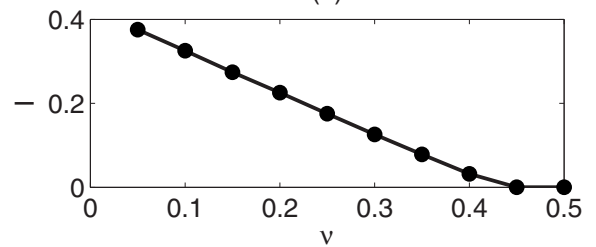

(b)

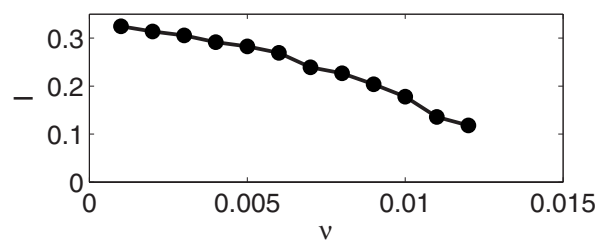

FIG. 5. Average infectives versus vaccine frequency $\nu$ for the SIRV model with $q=0.0016, r=0.002, p=0.003, A=0.1$. (SIRS parameters taken from [2].) (a) Static network. (b) Adaptive network, $w=0.04$. Curves are to guide the eyes.

non-Gaussian vaccine control methods. The model presented here extends and improves upon previous studies of homogeneous populations as in [27] and other adaptive network SIS models without vaccine control [1]. When the vaccination schedule was Poisson distributed, we found vaccination was far more effective in an adaptive network than a static one due to the interaction of vaccination and rewiring. Vaccine effectiveness was increased by two orders of magnitude for the parameters chosen for the SIV model and over an order of magnitude for the SIRV model. The network adaptation led to a higher average degree for susceptible nodes, and random vaccination of susceptibles exploited this heterogeneity. Further, the adaptive rewiring became more effective because of the presence of "safe" vaccinated nodes to rewire to.

The model classes we considered consisted of diseases in which there is no immune response (SIS) and diseases which produce only temporary immunity (SIRS.) Models with no immunity are suitable for many bacterial infections, such as meningitis, plague, and venereal diseases, as well as certain protozoan illnesses, such as malaria and sleeping sickness [35]. Temporary immunity models such as SIRS are appropriate for influenza [36], syphilis [37], cholera and typhoid fever [35], as well as pertussis [38]. Although not all of the above diseases currently possess a vaccine, research is ongoing to develop new vaccines. Future model and control variations might include host-vector modeling, seasonality, quarantine, and isolation, thus rendering the model more realistic for specific diseases.

In our class of SIS and SIRS models, we randomly vaccinated a fixed fraction $A$ of the susceptible nodes during each vaccination event. Information about the network structure was not required. This corresponds to the case where vaccine is inexpensive and anyone who is not infected can be vaccinated. The mean lifetime results indicate how the rate of epidemic extinction scales with vaccination frequency, providing information about the amount of vaccine needed. If the vaccine were in limited supply, it would be desirable to use some knowledge about the social network structure (such 
as the degrees) to target the vaccine more carefully. In that case, the vaccine pulses might be a fixed number of susceptibles rather than a fixed fraction. However, further study is needed to determine the optimal vaccination strategy. Another extension would be to adjust the rules for link evolution, such as to relax the assumption that nodes have full knowledge of others' disease status $[3,4]$.

\section{ACKNOWLEDGMENTS}

L.B.S. was supported by the Jeffress Memorial Trust, Army Research Office, Air Force Office of Scientific Research, and NIH (Award No. R01GM090204 from the National Institute of General Medical Sciences). I.B.S. was supported by the Office of Naval Research and the Air Force Office of Scientific Research.
[1] T. Gross, Carlos J. Dommar D’Lima, and B. Blasius, Phys. Rev. Lett. 96, 208701 (2006).

[2] L. B. Shaw and I. B. Schwartz, Phys. Rev. E 77, 066101 (2008).

[3] D. H. Zanette and S. Risau-Gusmán, J. Biol. Phys. 34, 135 (2008).

[4] S. Risau-Gusmán and D. H. Zanette, J. Theor. Biol. 257, 52 (2009).

[5] B. M. Bolker and B. T. Grenfell, Proc. R. Soc. London, Ser. B 251, 75 (1993).

[6] B. M. Bolker, IMA J. Math. Appl. Med. Biol. 10, 83 (1993).

[7] J. Patz, Proc. Natl. Acad. Sci. U.S.A. 99, 12506 (2002).

[8] D. Rand and H. Wilson, Proc. R. Soc. London, Ser. B 246, 179 (1991).

[9] L. Billings, E. M. Bollt, and I. B. Schwartz, Phys. Rev. Lett. 88, 234101 (2002).

[10] H. Andersson and T. Britton, J. Math. Biol. 41, 559 (2000).

[11] O. A. van Herwaarden and J. Grasman, J. Math. Biol. 33, 581 (1995).

[12] L. Allen and A. M. Burgin, Math. Biosci. 163, 1 (2000).

[13] J. A. Jacquez and C. P. Simon, Math. Biosci. 117, 77 (1993).

[14] V. Elgart and A. Kamenev, Phys. Rev. E 70, 041106 (2004).

[15] C. R. Doering, K. V. Sargsyan, and L. M. Sander, Multiscale Model. Simul. 3, 283 (2005).

[16] M. J. Keeling and P. Rohani, Modeling Infectious Diseases in Humans and Animals (Princeton University Press, Princeton, 2007).

[17] J. Verdasca et al., J. Theor. Biol. 233, 553 (2005).

[18] M. S. Bartlett, J. R. Stat. Soc. Ser. B (Methodol.) 11, 211 (1949).

[19] I. B. Schwartz, L. Billings, M. Dykman, and A. Landsman, J. Stat. Mech. 2009, P01005.

[20] A. Kamenev and B. Meerson, Phys. Rev. E 77, 061107 (2008).

[21] R. M. Anderson and R. M. May, Infectious Diseases of Humans: Dynamics and Control (Oxford Science Publications, New York, 1991).

[22] A. d'Onofrio, Math. Biosci. 179, 57 (2002).
[23] S. Gao, L. Chen, and Z. Teng, Bull. Math. Biol. 69, 731 (2007).

[24] B. Shulgin, L. Stone, and Z. Agur, Bull. Math. Biol. 60, 1123 (1998).

[25] L. Stone, B. Shulgin, and Z. Agur, Math. Comput. Modell. 31, 207 (2000).

[26] X. Wang, Y. Tao, and X. Song, Appl. Math. Comput. 210, 398 (2009).

[27] M. I. Dykman, I. B. Schwartz, and A. S. Landsman, Phys. Rev. Lett. 101, 078101 (2008).

[28] R. Pastor-Satorras and A. Vespignani, Phys. Rev. E 65, 036104 (2002).

[29] Z. Dezső and A.-L. Barabási, Phys. Rev. E 65, 055103(R) (2002).

[30] D. H. Zanette and M. Kuperman, Physica A 309, 445 (2002).

[31] J. Miller and J. M. Hyman, Physica A 386, 780 (2007).

[32] R. Cohen, S. Havlin, and D. ben-Avraham, Phys. Rev. Lett. 91, 247901 (2003).

[33] Y. Chen, G. Paul, S. Havlin, F. Liljeros, and H. E. Stanley, Phys. Rev. Lett. 101, 058701 (2008).

[34] L. M. Pacheco Santos, R. Paes-Sousa, J. Barbosa da Silva Junior, and C. Gomes Victora, Bull. World Health Organ. 86, 474 (2008).

[35] H. W. Hethcote, Math. Biosci. 28, 335 (1976).

[36] W. R. Derrick and P. van den Driessche, J. Math. Biol. 31, 495 (1993).

[37] N. C. Grassly, C. Fraser, and G. P. Garnett, Nature (London) 433, 417 (2005).

[38] H. W. Hethcote, SIAM Rev. 42, 599 (2000).

[39] Steady-state behavior occurs for other $q$ values, but we focus here on the oscillatory regions because the dynamics is more interesting. The qualitative advantage to including both vaccination and rewiring is the same whether or not oscillations occur.

[40] The bifurcation structure and infective levels depend primarily on the average vaccination rate $\nu A$ rather than on the frequency or amplitude individually. 